\title{
Prediction of Battery Behavior Subject to High-Rate Partial State of Charge
}

\author{
Patrick J. van Bree, Student Member, IEEE, André Veltman, Member, IEEE, \\ Will H. A. Hendrix, and Paul P. J. van den Bosch, Member, IEEE
}

\begin{abstract}
An online optimization procedure provides the parameters of a nonlinear battery model by taking into account a few minutes of measured current-voltage data. Within a defined range in terms of charge current, state of charge (SOC), and duration of charge and discharge events, the model is able to capture the relevant battery dynamics and predict the behavior for the next few minutes. From the battery behavior during specific events, the state of the battery can be revealed, which is defined as the state of function. Validation, which is carried out on measured current-voltage profiles, shows the accuracy of prediction during the high-rate partial SOC operation. Even with the data measured during a city drive within a microhybrid electrical vehicle, the method is able to predict the voltage level during high-rate discharge pulses (cranking).
\end{abstract}

Index Terms-Battery model, charge acceptance, high-rate partial state-of-charge (HRPSOC), hybrid electric vehicles (HEVs), lead-acid battery, state of function (SOF).

\section{INTRODUCTION}

$\mathbf{P}$ REDICTION of dynamic battery behavior is a critical issue for vehicle energy-management concepts that depend on the battery as an energy buffer [1]. In hybrid electric vehicles (HEVs), the production of electrical energy is scheduled [2]. During phases when it is economically interesting (e.g., deceleration), boost charging is applied [3]. Energy consumption by the electric power net takes place when the production is uneconomic (e.g., during acceleration or standstill periods). One of the preconditions for this type of use is the ability of the battery to accept charge; the battery is, therefore, continuously operated in a partial state-of-charge (PSOC) (e.g., $70 \%<$ SOC $<90 \%$ ). The intended use, further indicated as high-rate PSOC (HRPSOC) [4], brings along shallow discharge cycles; $<10 \%$ depthof-discharge (DOD) [3]. The accumulated ampere-hour (Ah) turnover can, however, be significant. The involved charge and discharge currents can be several times higher (high rate) than the battery's C-rate.

\section{STATE OF FUNCTION}

The research goal is to predict the battery's current-voltage relation with specific charge or discharge events. The battery

Manuscript received November 13, 2007; revised May 19, 2008 and June 22, 2008. First published June 27, 2008; current version published February 17, 2009. This work was supported in part by Ford Research Center Aachen. The review of this paper was coordinated by Mr. D. Diallo.

The authors are with the Control Systems, Department of Electrical Engineering, Eindhoven University of Technology, 5600 Eindhoven, The Netherlands (e-mail: P.J.v.Bree@ tue.nl).

Digital Object Identifier 10.1109/TVT.2008.928005

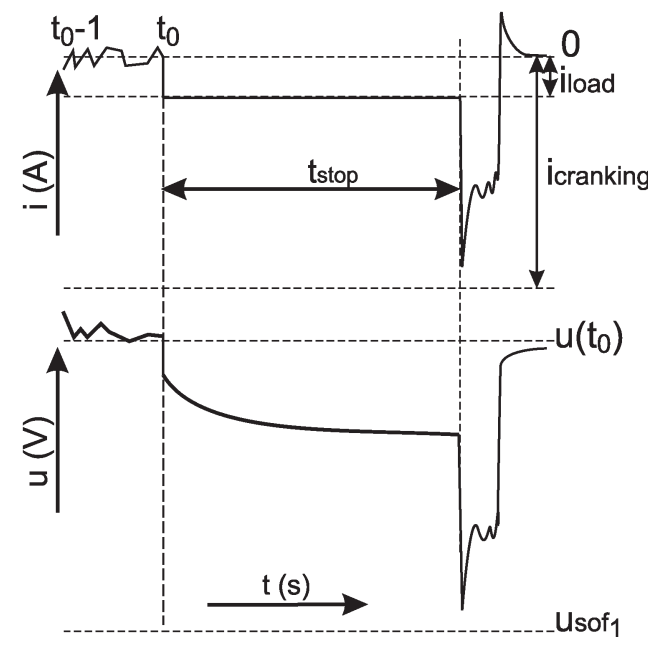

Fig. 1. Cranking capability $\mathrm{SOF}_{1}$. From $t=t_{0}$, the battery is discharged with current $i_{\text {load }}$ for $t_{\text {stop }}$ seconds. Immediately after the discharge period, a highrate discharge pulse, representing cranking of the combustion engine, is applied. The actual duration of a cranking pulse can vary from less than half a second to a few seconds, whereas $t_{\text {stop }}$ can be several minutes.

management system can use this information to adapt its strategy. The nature of the defined events is related to the HEV application, and the response reveals whether the battery can still fulfill a certain duty. The battery management system compares the predicted values with the required ones and decides if the current energy management strategy should be updated or not. The approach of considering the functionality derived from the transient behavior is defined as the state of function (SOF). A few examples will illustrate the relation between SOF, state of charge (SOC), and state of health $(\mathrm{SOH})$.

The capability to crank the combustion engine after several minutes of engine-off (e.g., traffic light, bridge) can be derived from the minimum voltage reached during the simulation of continuous discharge followed by a cranking pulse. An illustration of the cranking capability $\left(\mathrm{SOF}_{1}\right)$ is provided in Fig. 1 $\left(i_{\text {load }} \in[0,-100]\right.$ A for $t_{\text {stop }}$ seconds, $i_{\text {cranking }} \in[-100$, $-300]$ A for $\left.t_{\text {cranking }} \in[0.5,3] \mathrm{s}\right)$. In this case, the minimum voltage reached $\left(u_{\mathrm{SOF}_{1}}\right)$, occurring during the cranking pulse, is compared to a predefined voltage level required by the starter engine to crank the combustion engine (e.g., 7.2 V). The quantities $i_{\text {load }}, i_{\text {cranking }}$, and $t_{\text {stop }}$, and the minimum $u_{\mathrm{SOF}_{1}}$ follow from the real-life application.

In other words, the goal is to predict if the battery is still capable of delivering the required power to crank the combustion engine after a defined period of discharge. An extension to $\mathrm{SOF}_{1}$ is to predict the maximum duration of discharge $t_{\text {stop }}$ 

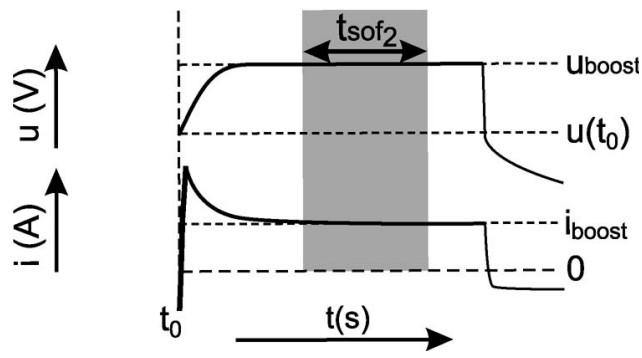

Fig. 2. Charge acceptance $\mathrm{SOF}_{2}$. At $t=t_{0}$, boost charging is applied; the voltage is raised to the $u_{\text {boost }}$ set point. The gray area indicates the defined period $t_{\mathrm{SOF}_{2}}$ over which the current is averaged to obtain $i_{\text {boost }}$.

when the voltage during the cranking event is allowed to drop to a defined $u_{\mathrm{SOF}_{1}}$.

The charge acceptance $\mathrm{SOF}_{2}$ is a battery requirement that determines the charge balance of the power supply system [5] and provides the response of the battery during boost charging [3]. Charge acceptance is defined as the average current flowing into the battery $\left(i_{\text {boost }}\right)$ over a defined period $t_{\mathrm{SOF}_{2}}$ of time when forcing a constant overvoltage (Fig. 2). The predicted charge acceptance gives a good indication if the battery is allowed to be (dis)charged.

In the first instance, the described SOF definitions (i.e., cranking capability and charge acceptance) are only valid with short-term use. Both consider the battery's transient behavior for the next couple of minutes. If rest periods are involved, the dynamic (transient) behavior will change even if the battery is kept at a constant SOC and temperature. For example, cranking after engine stop-start at a traffic light during a city drive in the afternoon will not be an accurate representation of the first cranking pulse in the next morning even if the SOC and temperature are exactly the same. The prediction for long-term situations is not taken into account in this paper.

An accurate online identification of SOH and SOC within (hybrid) electrical vehicles is still a booming topic of research; a clear indication that it is very hard to achieve the accuracy required by energy management systems [6], [7]. SOF is highly related to the combination of $\mathrm{SOC}, \mathrm{SOH}$, and temperature $(T)$. A combination of a low temperature and a low SOC will always result in a relatively low voltage during cranking. On the other hand, a large charge acceptance indicates a low SOC. However, the transient behavior of the battery with a defined combination of SOC, $\mathrm{SOH}$, and $T$ is not unique, since, for instance, after rest periods the behavior will be different. The examples illustrate that when the dynamic behavior can accurately be predicted, and the desirable behavior is known (with specific events), this information can be used as input for battery management. In any case, battery management depends on a number of models and algorithms since comprehensive battery state observers do not exist.

\section{MODEL-BASED APPROACH}

The transient current-voltage behavior is predicted by a battery model of which the parameters are established online using a few minutes of real-life driving data (current and voltage). No extra tests are required, and the whole procedure is based

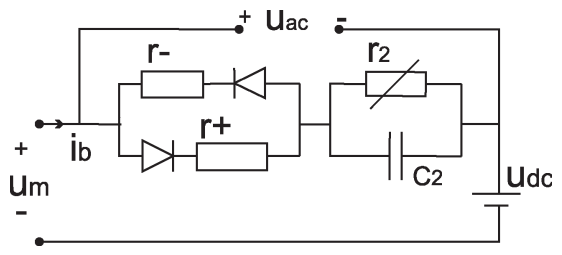

Fig. 3. Nonlinear battery model as used for online short-term SOF prediction.

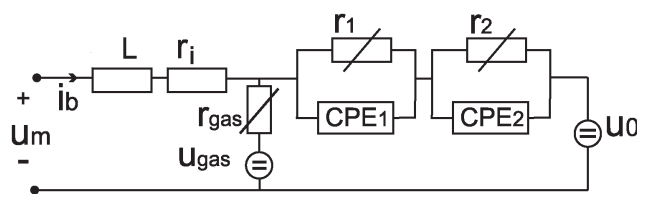

Fig. 4. Impedance-based nonlinear simulation model for a VRLA battery [9], [10].

on the available data. The method should detect segments of data suitable for identification. By identification of the model's parameters, for instance, every $10 \mathrm{~min}$, the model adapts to a possible changed situation in terms of SOC and temperature. Variations of these quantities are considered to be insignificant within the 10-min interval. The need for a temperature- or SOCdependent model is avoided this way.

The SOF approach works with any type of model as long as it is able to capture the dynamics involved with the application. There is no explicit need to fix the model structure or to use a physically based model. However, the model parameters have to be established online using real-life vehicle data, which put bounds on complexity. The presented model, which is used for SOF prediction (Fig. 3), is a simplification of an existing impedance-based nonlinear simulation model (Fig. 4). To guarantee (fast and robust) convergence of the optimization procedure, the model structure is fixed and depends on as few parameters as possible.

\section{A. Identification Data}

An average drive takes less than $30 \mathrm{~min}$ (and seldom more than $3 \mathrm{~h}$ ) [8]. Suitable model parameters should, therefore, be identified within the first $5-10 \mathrm{~min}$ for the method to have added value. This already puts bounds on the amount of data used for identification. Next to that, the fewer the data (number of samples), the faster the optimization procedure. The number of samples depends on the sampling interval and the length of the used window. The most critical lower bound on the sample frequency comes from the cranking pulses. If the exact minimum voltage is required and the cranking pulse has a duration of $0.5 \mathrm{~s}$, a sample frequency of $100 \mathrm{~Hz}$ is sufficient.

The dynamic range of a battery is enormous; it varies from microseconds to hours, days, and years. Here, it is important that with only a few minutes $(2-6 \mathrm{~min})$ of data, an accurate prediction of the next coming tens of minutes (10-40 $\mathrm{min})$ is obtained. The optimal duration of window used is not under question. It should be proved that it is possible to use several minutes for the identification and to predict for the next half hour. 


\section{B. Impedance-Based Nonlinear Simulation Model}

The model structure of the impedance-based nonlinear simulation model [9] (Fig. 4) is established with the help of electrochemical impedance spectroscopy (EIS) applied to valve-regulated lead-acid (VRLA) batteries. Out of a collection of impedance spectra obtained over a wide working range of temperature $(T)$, current $(I)$, and SOC, the model structure and parameterization are established. The impedance spectra are obtained from the battery's response to an ac current (microcycle) superimposed on a dc current $\left(I_{\mathrm{dc}}\right)$. The amplitude of the microcycle is so small that the battery behavior can be considered linear in the working area of current, temperature, and SOC. The presented measurements in [9] are carried out over a grid of $T=[-25,40]{ }^{\circ} \mathrm{C}, I_{\mathrm{dc}}=[-10,10] \mathrm{A}$, and $\mathrm{SOC}=$ $[20,90] \%$. Measuring a single impedance spectrum (constant SOC, $T$, and $I_{\mathrm{dc}}$ ) takes about $1000 \mathrm{~s}$; the model's bandwidth is $[1 \mathrm{mHz}, 6 \mathrm{kHz}]$. The variation of the model parameters with SOC and $T$ is stored in lookup tables. The presented approach can be used for other battery types (different architecture, chemistry, etc.), but possibly, a slightly different model structure is required.

Excitation of the simulation model with typical HRPSOC profiles showed that the influence of $\mathrm{CPE}_{1}$ is not significant, and it is, therefore, neglected. The value of the ohmic series resistance $r_{i}$ (Fig. 4) is added as an offset to $r_{1}$. As described in [9] and [10], the resistance $r_{1}$ has charge and discharge modes with a smooth transition that can slightly be asymmetric to zero current. To reduce the number of parameters involved, a piecewise linear approach results in a separate charge $r_{+}$ and discharge $r_{-}$resistance in combination with ideal diodes (Fig. 3).

The charge transfer resistance $r_{2}$ depends on the voltage across the capacitive element in parallel and is described by the Butler-Volmer relation

$$
i_{r 2}(\mathbf{u})=I_{0}\{\exp (\alpha \beta \mathbf{u})-\exp ((\alpha-1) \beta \mathbf{u})\}
$$

The implemented equation [9], [10] considers the three parameters $I_{0}, \alpha$, and $\beta$.

In the frequency band of interest (approximately $10^{-3}$, $10^{2} \mathrm{~Hz}$ ), the impedance of the inductance $L$ is not significant. Therefore, $L$ has been neglected.

The Constant Phase Elements (CPEs) in Fig. 4 are semidissipative-capacitive elements. In the frequency domain, the impedance (voltage divided by current) of the CPE is described by

$$
Z_{\mathrm{CPE}}(j \omega)=A \cdot(j \omega)^{-p}
$$

The power $p$ can have a noninteger value, which implies a very large bandwidth (infinite memory in time). Where a capacitor $(p=1)$ introduces a constant phase shift of $-90^{\circ}$, the phase of a CPE will shift by $-90^{\circ} \cdot p$. In a physical approach, a capacitor only stores energy where a CPE also consumes energy. A resistance $(p=0)$ does not introduce any phase shift. The typical values involved with batteries are $0.5<p<1$.
The mathematical basis of these noninteger (or fractional) integrators is found in [11]. In addition to electrochemical applications like batteries (electrode-electrolyte interface) and fuel cells (diffusion with proton membrane exchange fuel cells [12]), this type of behavior is among others observed with viscoelasticity and dielectric relaxation [13].

To reduce the number of parameters involved and to simplify the overvoltage dependence of the nonlinear resistance $r_{2}$ in parallel, the dissipative behavior of $\mathrm{CPE}_{2}$ is neglected, and it is replaced by a single capacitor $\mathrm{C}_{2}$. The results obtained with different implementations of the CPE approximations and the parallel nonlinear resistance-CPE branch are not described in this paper. Information about numerical simulation and discrete approximation schemes is found in [14] and [15].

The open-circuit voltage model $u_{0}$ (Fig. 4), as presented in [9], only depends on the SOC. Identification using EIS is limited in precision since the measurement time during model parameterization is limited to assure a quasi-stationary state of the battery. Only looking at the presented SOC-dependent $u_{0}$ model and the defined short-term high-rate partial SOC (HRPSOC) use, considering $u_{0}$ as a constant value is valid as long as the SOC does not vary significantly.

In [16], $u_{0}$ is replaced by a voltage source that takes into account the acid concentrations in both electrodes. These are calculated by an electrolyte transport model. The local acid concentration is influenced by diffusion, change of porosity, and acid generation. The presented measurements and simulations show that electrolyte transport can be observed within tens of minutes. The possibilities for the implementation of these model improvements in the model for online prediction have yet to be studied.

The side reactions that occur during charge and overcharge are represented by the gassing branch $\left(r_{\text {gas }}, u_{\text {gas }}\right)$. The parameters and structure of the gassing branch, as presented in [9], do not accurately describe the true behavior. Because the validity of the gassing branch is uncertain, and because gassing does not occur in normal battery use, it is neglected in the battery model used for online prediction.

The charge acceptance with HRPSOC [4] following from the nonlinear impedance-based simulation model is, however, much too optimistic [17]. It is stated that with shallow cycling the charge acceptance increases with the increased cycling time, although the SOC is approximately stable. Improved models take into account the short-term history of the battery current. The accumulation and depletion of the dissolved $\mathrm{Pb}^{2+}$ ions are described and identified as the main limiting effect during dynamic charging [18].

In [17], the gassing branch is replaced by an overcharge model. Oxygen evolution at the positive electrode, hydrogen evolution at the negative electrode, and oxygen reduction at the negative electrode are taken into account.

\section{Nonlinear LeAst-SQuare Optimization}

The model parameters are obtained by minimizing a cost function using the iterative nonlinear least-square LevenbergMarquardt optimization method [19]. Due to the physical representation of the model, the upper and lower bounds for 
the parameters can be derived (e.g., no negative resistances). Parameter values that are beyond the bounds are penalized. In between the bounds, the penalty function is equal to zero. Outside the bounds, the additional cost exponentially increases.

The defined cost function $E(\theta)$ considers the sum of squares of the difference between the measured battery voltage and the simulated model output

$$
\begin{aligned}
E(\theta) & =\sum_{n=2}^{N}\left[W(n) \cdot\left(u_{b}(n)-u_{m}(\theta, n)\right)\right]^{2} \\
& =\sum_{n=2}^{N}[W(n) \cdot e(\theta, n)]^{2} .
\end{aligned}
$$

Here, $\theta$ represents the tunable parameters of the model. The method is gradient based; it makes use of the Jacobian matrix and the error vector $e(\theta)$ to determine its search direction and step size.

The fixed model structure makes it possible to derive an analytic expression for the Jacobian matrix. This significantly speeds up the optimization procedure (in comparison with a finite-difference approach). Next to computation time, analytic expressions are more accurate.

An iterative optimization procedure needs initial values (a first guess) for the optimization parameters. Since the physical order of magnitude of the model parameters varies a lot (approximately $10^{-3}$ to $10^{3}$ ), the initial parameter values are normalized to 1 . The difference in magnitude would otherwise slow down the convergence of the optimization. The physical values are obtained by multiplication of the tunable parameters $\theta$ with their order of magnitude.

To emphasize important fragments of data, the residual $e(\theta, n)$ is multiplied with a weighting vector $W(n)$ [(3)]. For instance, the number of samples involved with high-rate discharge pulses is relatively low compared with the total. Therefore, $e(\theta)$ is emphasized with these events. $W(n)$ is normally equal to 1 , except for high-rate discharge pulses where it is equal to 10. The choice for the weighting value is application dependent.

Instead of introducing the constant $u_{\mathrm{dc}}$ (Fig. 3) as a parameter in optimization, it is defined as the mean of the difference between $u_{b}$ and $u_{\text {ac }}$. In other words, the error vector is defined as the difference of the measured voltage and the dynamic model voltage minus the mean of this difference, i.e.,

$$
\begin{aligned}
u_{m}(\theta, n) & =u_{\mathrm{ac}}(\theta, n)+u_{\mathrm{dc}}(\theta) \\
u_{\mathrm{dc}}(\theta) & =\frac{1}{N} \sum_{n=2}^{N}\left[u_{b}(n)-u_{\mathrm{ac}}(\theta, n)\right] .
\end{aligned}
$$

The more that the dynamic behavior of measurement and model match, the more optimal the value of $u_{\mathrm{dc}}$. An initial value for the first iteration is, however, required.

The simplified series model contains only one state, i.e., the voltage across capacitor $C_{2}$. During each iteration, $C_{2}$ 's initial
TABLE I

Initial Parameter VALUes Along With CoRresponding UPPER AND LOWER BOUNDS

\begin{tabular}{llll}
\hline parameters & magnitude & $\begin{array}{l}\text { lower } \\
\text { bound }\end{array}$ & $\begin{array}{l}\text { upper } \\
\text { bound }\end{array}$ \\
\hline tunable & & & \\
\hline$r_{-}$ & $0.008 \Omega$ & $0.001 \Omega$ & $0.1 \Omega$ \\
$r_{+}$ & $0.010 \Omega$ & $0.001 \Omega$ & $0.1 \Omega$ \\
$C$ & $1000 \mathrm{~F}$ & $100 \mathrm{~F}$ & $5000 \mathrm{~F}$ \\
$\beta$ & $20 \mathrm{~V}^{-1}$ & $1 \mathrm{~V}^{-1}$ & $1000 \mathrm{~V}^{-1}$ \\
\hline fixed & & & \\
\hline$I_{0}$ & $1 \mathrm{~A}$ & & \\
$\alpha$ & 0.5 & & \\
\hline$\left.u_{d c}\right|_{n=1}$ & 12 & & \\
\hline
\end{tabular}

voltage is set such that the model and measured voltages are equal at $n=1$, i.e.,

$$
\begin{aligned}
& \left.u_{C_{2}}\right|_{n=1}=u_{b}(n)-u_{r_{ \pm}}(n)-u_{\mathrm{dc}} \\
& \text { if } i_{b}<0 \\
& u_{r_{ \pm}}=i_{b}(n) \cdot r_{-}
\end{aligned}
$$

else

$$
u_{r_{ \pm}}=i_{b}(n) \cdot r_{+}
$$

end.

The sum of squares in (3) is, therefore, taken from $n=2$ to the total number of samples $N$.

\section{VALIDATION}

To show the potential of the SOF approach in combination with the nonlinear battery model, the procedure is tested on three data sets, including real-life vehicle data. The currentvoltage profiles of the first two sets result from laboratory experiments and are carried out using a controlled load and power source. The third set contains real-life vehicle data obtained during a city drive using the Ford Fiesta microhybrid technology demonstrator. The batteries under test are the Exide Technologies VRLA with orbital topology $(12 \mathrm{~V}, \mathrm{C} 20=$ $50 \mathrm{Ah}, \mathrm{CCA}=800 \mathrm{~A}$ ). It should be mentioned that all the measurements took place within a (environmental) temperature range of $[15,25]^{\circ} \mathrm{C}$.

The presented current and voltage signals are sampled at $100 \mathrm{~Hz}$. The original data were sampled at $1 \mathrm{kHz}$ and prefiltered using an eight-order Bessel-type anti-aliasing filter having a cutoff frequency that is well below half the Nyquist frequency. This specific filter structure preserves the wave shape (in exchange for steepness). For an accurate determination of the lowest voltage during cranking $\left(\mathrm{SOF}_{1}\right)$, the relatively high sample frequency and the advanced filter type are essential. The data obtained without anti-aliasing or data filtered by moving average filters result in the instability of the solver of the ordinary differential equation (ODE).

Table I shows the initial values for the iterative procedure. Both parameters $I_{0}$ and $\alpha$ are fixed in all tests. This setting 

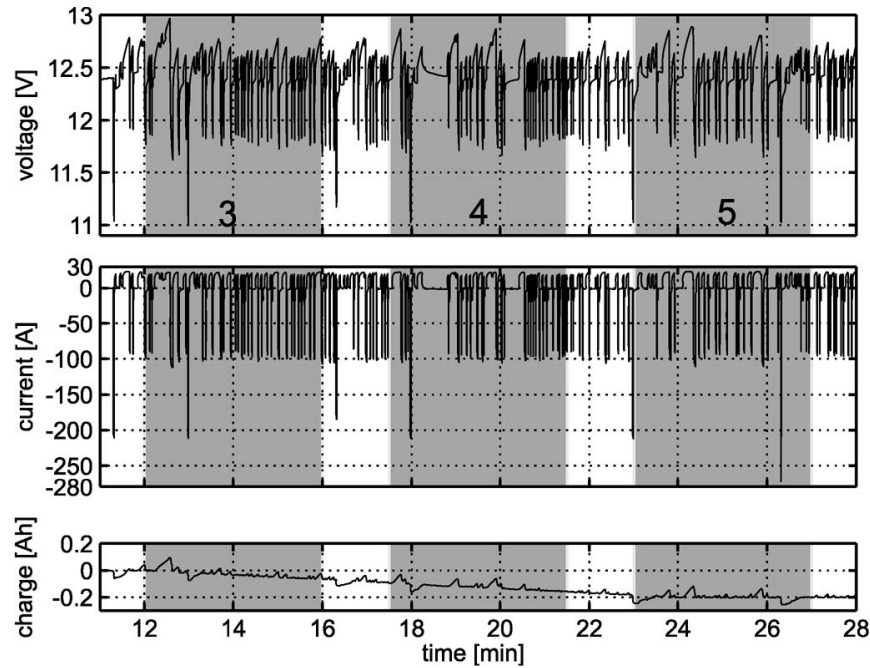

Fig. 5. Data set 1. Measured (upper) voltage and (middle) current profiles The lower plot denotes the charge difference in ampere-hour (the charge signal is set to zero in the beginning of the measurement). The gray rectangles denote three of the 11 different identification windows (numbers 3, 4, and 5). All windows are shifted $5.5 \mathrm{~min}$ and take 4 min each. $[1-5,6.5-10.5,12-16, \ldots$, 56-60] $\mathrm{min}$. The total measurement takes $60 \mathrm{~min}$.

resulted in the best prediction quality to number of parameter ratio. The number of model parameters is equal to seven, of which four are tunable (Table I), and $u_{\mathrm{dc}}$ is a result of optimization [see (4)]. The weighting vector $W(n)$ is equal to 1 , except for discharge currents of more than $I=-150 \mathrm{~A}$, and $W$ is equal to 10 . The number of iterations is limited to 20 .

\section{A. Data Set 1, Laboratory Test 1}

A rapidly alternating sequence of charge-discharge events is programmed into a hardware-in-loop setup. To simulate the engine stop-start feature of HEVs, pulses appear every few minutes ([-150, -300] A for $1 \mathrm{~s})$. Each event takes several seconds, and the SOC of the battery is, however, not significantly changing $(<1 \%)$. To make sure that the secondary reactions during charging have a minor influence, the maximum charge voltage and current are limited.

In Fig. 5, 17 of the total of $60 \mathrm{~min}$ are shown. The three gray areas denote identification window numbers 3,4 , and 5. In total, 11 windows of $4 \mathrm{~min}$ each, shifted by $1.5 \mathrm{~min}$, are established: $[1-5,6.5-10.5,12-16, \ldots, 56-60] \mathrm{min}$.

In the first instance, the performance of the method is determined by the inspection of the difference between model and measurement. Simulation is carried out over [0-60] min. A comparison of measurement and prediction, from 40 to $50 \mathrm{~min}$, is provided in Fig. 6. Here, the parameters that are established using window 3 are used.

The correspondence between the measurement and the model (error smaller than $\pm 0.2 \mathrm{~V}$ ), with model parameters established $25 \mathrm{~min}$ in advance, shows the validity of the approach. From this, it is concluded that the dynamic behavior does not change within tens of minutes of rapidly alternating between charging and discharging. The model structure and the identification procedure are considered valid for the presented range of excitation.
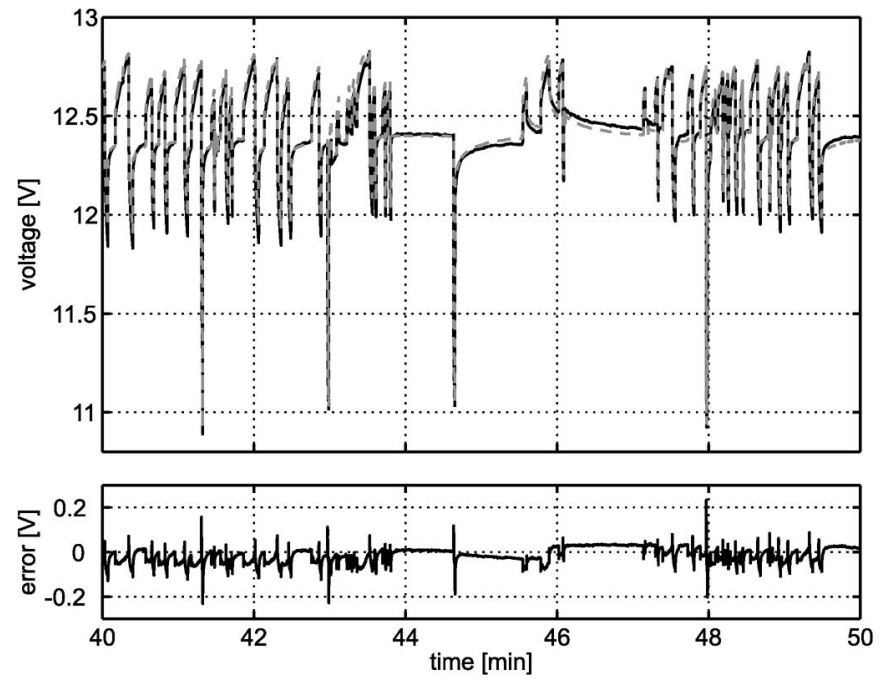

Fig. 6. Data set 1. Correspondence [40-50] min between (dashed gray) model and (solid black) measurement using the model parameters established on identification window 3, [12-16] min. The error between both is shown in the lower plot.

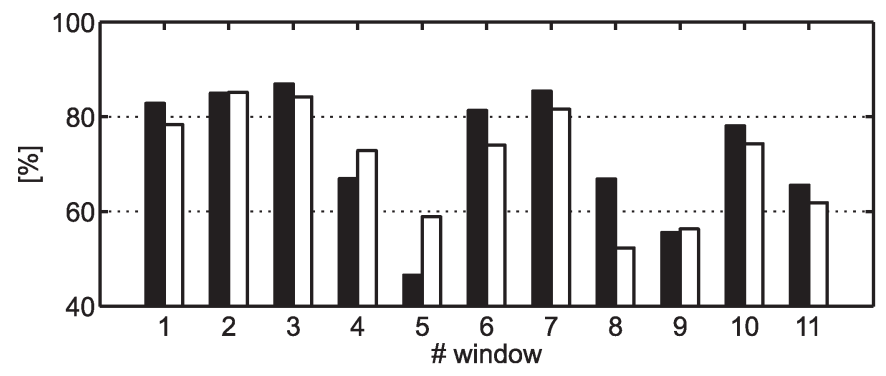

Fig. 7. Data set 1. Correspondence of the dynamic behavior in percentage as formulated in (6). The results are presented for the different windows. The black bars denote the identification windows, and the white bars denote the validation windows.

In (6), the $l_{2}$ norm of the error signal is divided by the $l_{2}$ norm of the measured dynamic voltage (offset removed). This way, the quality parameter $Q_{\text {dyn }}$ expresses the ratio between the error and the dynamic excitation in percentage as follows:

$$
Q_{\mathrm{dyn}}=100 \% \cdot\left(1-\frac{\sqrt{\sum e^{2}}}{\sqrt{\sum\left(u_{b}-\overline{u_{b}}\right)^{2}}}\right) .
$$

The $Q_{\mathrm{dyn}}$ criterion is applied to the error between the measurement and the model over the whole 60 min using the parameters established on different windows (Fig. 7). For comparison, the criterium is also applied to the identification windows (4 min) themselves. The state of the battery is considered constant since the temperature and SOC do not significantly differ.

The parameters established on windows $1,2,3,6$, and 7 provide a $Q_{\text {dyn }}$ higher than $80 \%$, but for windows 5 and 9 , $Q_{\mathrm{dyn}}<60 \%$. Comparing the excitation for different windows (windows 3, 4, and 5 in Fig. 5), it is more or less the same. However, the results illustrate that the obtained parameters are not equal; there is a difference of more than $30 \%$ between windows 3 and 5, for instance. However, both contain a high-rate discharge pulse and are subject to the applied weighting [see (3)]. When the $Q_{\mathrm{dyn}}$ percentage is low for the 


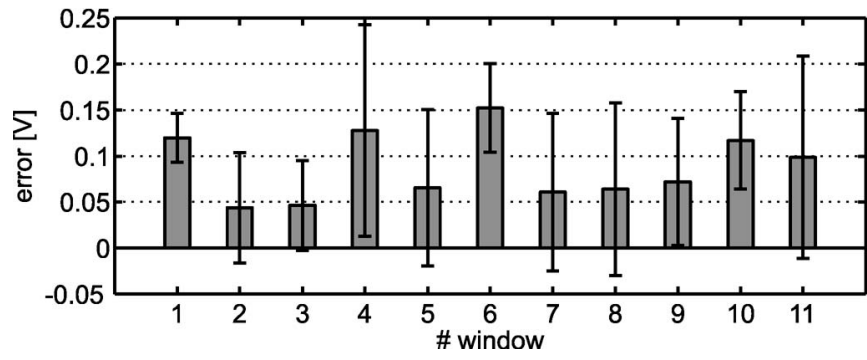

Fig. 8. Data set 1. Average of the absolute error and corresponding standard deviations over 14 cranking pulses within the set of $60 \mathrm{~min}$.

identification itself, the score for the validation will also be low. The same holds for good scores; if $Q_{\text {dyn }}>80 \%$ with the identification window, then the validation has similar scores.

Considering the cranking capability $\mathrm{SOF}_{1}$, only the absolute lowest voltage during high-rate discharge pulses $(\leq 150 \mathrm{~A})$ is important. Figs. 5 and 6 provide the current-voltage and error between the model and measurement. In $60 \mathrm{~min}, 14$ pulses occur. The error with the pulses may seem very large (up to $0.2 \mathrm{~V}$ for window 3 ), but in fact, these large errors appear at the falling and rising edges of the high-rate pulses, and the error at the absolute lowest voltage is a lot less. To illustrate this, Fig. 8 provides the average absolute error (for the 14 pulses) and the corresponding standard deviation. For window 3, the average absolute error is less than $0.05 \mathrm{~V}$. Note that this is one of the characteristics of the SOF approach. The prediction of the dynamic behavior needs to be accurate for the SOFtest only. The absolute minimum is important, and the edges are not.

Both criteria are quite different, but the nature of excitation in all the windows is the same. The hypothesis was, therefore, that the results for different windows were comparable, but they are not. The reason for this is not found. No indicator, which can provide a measure for the prediction quality in advance, is found. Without actual predicting, the most appropriate data for optimization could not be established. Studies [for instance, the influence of the presence of specific events (cranking pulses, longer continuous discharge), number of iterations required, window length, quality of dynamic match $Q_{\mathrm{dyn}}$, or the final or initial sum of squares $E(\theta)$ ] did not reveal the desired indicator.

The results for window 3 (for both $Q_{\mathrm{dyn}}$ and the error with discharge pulses) show that it is possible to predict the battery behavior with an accuracy of about $90 \%$ (dynamic voltage range $\approx 2 \mathrm{~V}$, maximum error $\approx 0.2 \mathrm{~V}$ ). However, the quality of prediction with the presented model structure is highly sensitive to the parameters. Unfortunately, the requirements for a suitable identification window are not revealed.

\section{B. Data Set 2, Laboratory Test 2}

The battery is excited with a similar profile as in data set 1 , but longer continuous charge events occur in the second half of data set 2 (Fig. 9). At the end, a discharge period of about $1 \mathrm{~min}$ is applied (Fig. 10). The discharge current is increased to $I=-130$ A. The change in SOC is within $1 \%$. The model parameters are established on 4 min of data [27-31] min. Before and within this window, there were no long charge events.
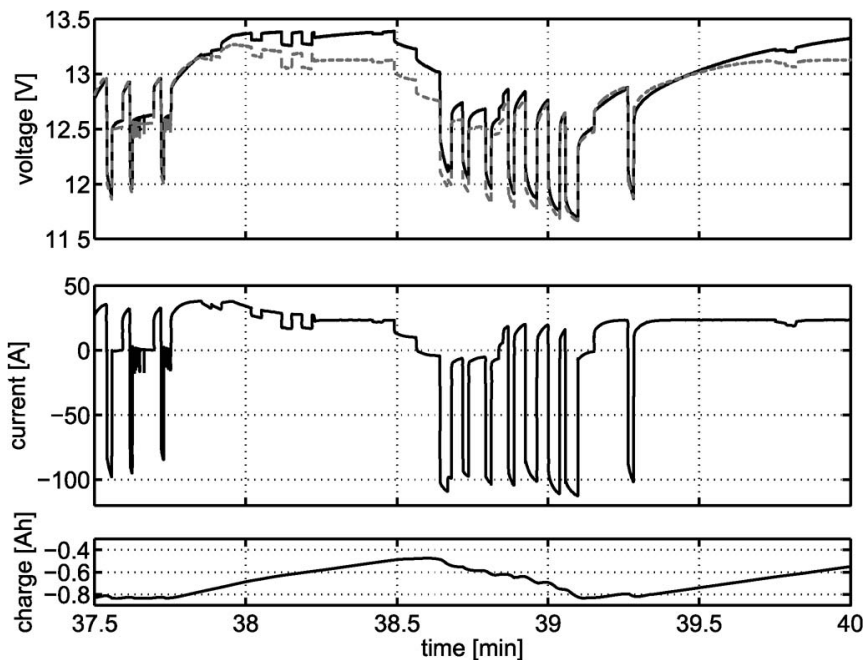

Fig. 9. Data set 2. Correspondence [37.5-40] min between (dashed gray) model and (solid black) measurement. The total validation took from 31 to $60 \mathrm{~min}$. Identification was carried out on $4 \mathrm{~min}$ of data, [27-31] $\mathrm{min}$. The lower plot shows the charge level that is set to zero at the beginning of the measurement $(0 \mathrm{~min})$

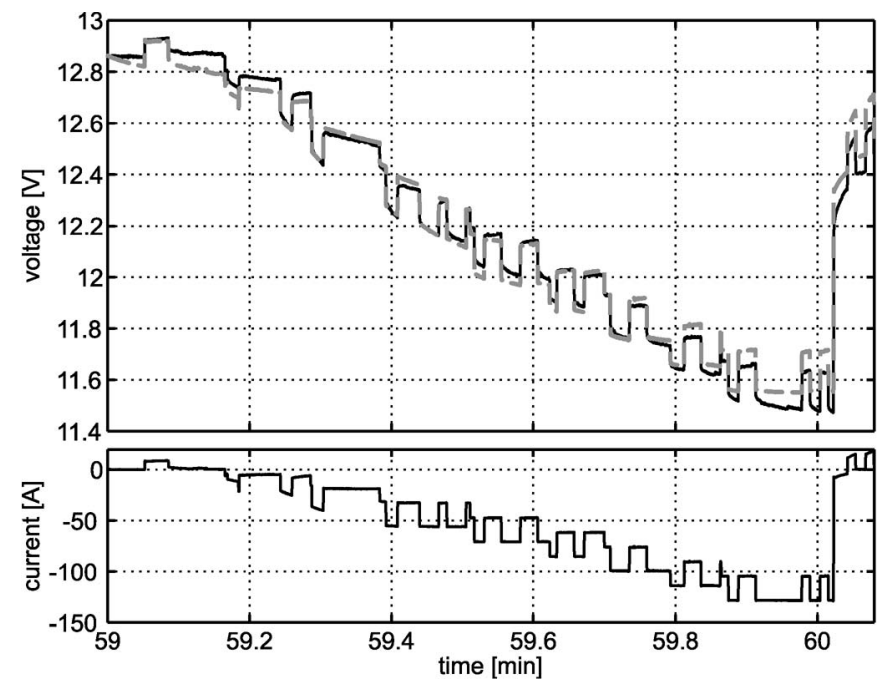

Fig. 10. Data set 2. Correspondence between (dashed gray) model and (solid black) measurement for a discharge event that lasts almost a minute. The discharge current increases to about $I=-130$ A. Prediction starts at $59 \mathrm{~min}$, and the identification window is again [27-31] $\mathrm{min}$.

1) Charging: Studying the comparison between the model and the measurements during charging (Fig. 9), it is observed that the predicted voltage is too low. For the prediction of the charge acceptance, this would mean that the predicted current is way too high (a fixed voltage would be taken as input). In [17] and [18], the shortcomings of the presented model structure with charging are explained.

The model shows large deviations for charging, but it is important to notice that after charging the model still accurately describes the behavior (around $39 \mathrm{~min}$ ). The longer charge events (in this case more than $20 \mathrm{~s}$ ) do not influence the battery behavior for current profiles rapidly alternating between charge and discharge. This experiment is a clear illustration that the presented model structure needs to be extended. 

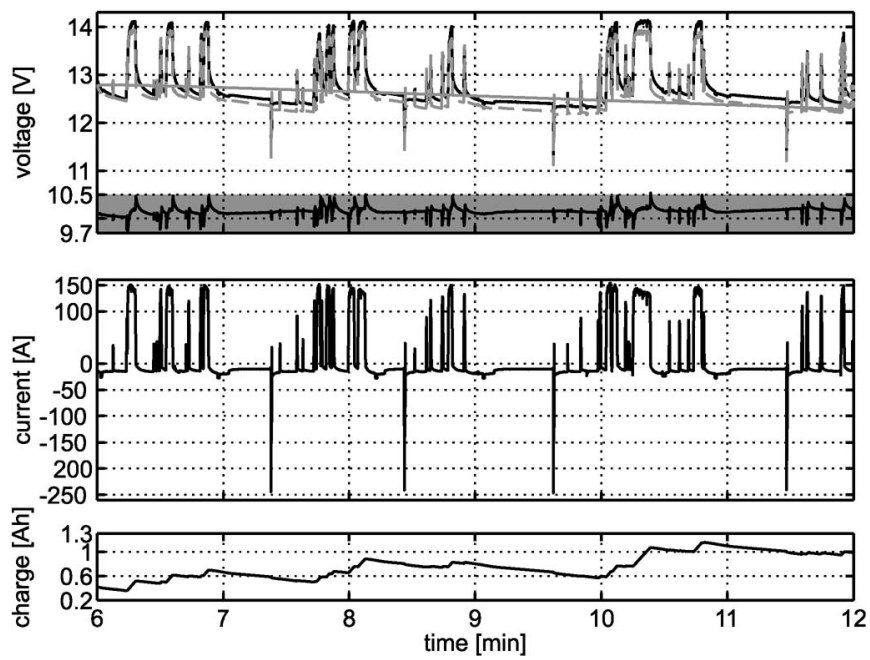

Fig. 11. Data set 3. Correspondence [6-12] min between model (dashed gray) and measurement (solid black) for real-life vehicle data. The model parameters are identified on the first 6 min of the data. (Solid black) The error is situated around $10 \mathrm{~V}$. The error bounds are indicated by the gray rectangle [9.7-10.5] V.

2) Discharging: For the prediction of $1 \mathrm{~min}$ of high-rate discharge, the same parameter set is used (established on [2731] $\mathrm{min}$ ). The discharge current is increased to $I=-130 \mathrm{~A}$. At the start of prediction (59 min), the measured and model voltages are matched [see (5)]. The error is at a maximum of $0.15 \mathrm{~V}$. It should be noted that the discharge current is about three times as high as with the presented charging profiles. Although there is only a single long discharge event shown, it is concluded that the prediction for discharging is much more accurate than during charging.

\section{Data Set 3, Real-Life Vehicle Test}

The presented test drive took place in the city of Aachen, Germany, using a micro-HEV. The Ford Fiesta 1.4-L gasoline, featuring regenerative braking and engine stop/start operation, has a single-battery $14-\mathrm{V}$ powernet topology. With microhybrids, no electric propulsion is involved; it is a conventional vehicle that uses the (lead-acid) battery as an energy buffer. The battery is situated in a battery case within the engine compartment. Within the measured $20 \mathrm{~min}$, the battery is charged with $\approx 1$ Ah $(\approx 2 \%$ SOC $)$. The presented real-life vehicle data (Fig. 11) involve higher charge voltages $(\approx 14.2 \mathrm{~V})$ than those presented in data sets 1 and $2(13,13.5 \mathrm{~V})$.

The model parameters are established on the first $6 \mathrm{~min}$ of data set 3. The prediction for the next 6 min is shown in Fig. 11. The upper plot also provides the error, which is situated around $10 \mathrm{~V}$, and the error bounds are $[-0.3,0.5] \mathrm{V}$. The identification window itself contains five cranking pulses, which can explain the accurate results with these events (average absolute error of $0.1 \mathrm{~V}$ and standard deviation of $0.05 \mathrm{~V}$ ).

The largest errors are observed during charging. The total error signal has an offset up from the first charge event; most likely, the unmodeled secondary reactions are compensated by an incorrect parameter setting (which provided the minimum sum of squares).
The error during charging (up to $0.5 \mathrm{~V}$ ) makes the model useless for the prediction of charge acceptance (predicted currents would be way too high). However, the results show that the model and procedure are suitable for the prediction of the cranking capability.

\section{CONCLUSION}

The SOF-based approach has shown that the comparison of the desired and predicted dynamic behaviors can provide a similar information to the battery-management system as the combination of SOC, SOH, and $T$ does. SOF indicates the ability of the battery to fulfil a certain duty. From the response with characteristic events, the state of the battery can be derived.

The SOF method can be used with any model that captures the dynamic current-voltage relation of the battery. The applicability depends on the accuracy of prediction and the possibility to establish the optimal model parameters online.

For online prediction during the HRPSOC use of a VLRA battery within a micro-HEV, an existing impedance-based nonlinear simulation model is simplified. By making use of the fixed model structure, an analytic expression for the Jacobian matrix is obtained. This significantly speeds up the optimization and is beneficial for accuracy.

Validation using both real-life vehicle data and laboratory tests shows that the model structure can accurately describe the current-voltage relation with profiles rapidly alternating between charging and discharging. With an accuracy of $\pm 0.2 \mathrm{~V}$, an error $<10 \%$ can be obtained under the conditions that the voltage and duration of charge are limited $(<13.5 \mathrm{~V},<10 \mathrm{~s})$. The accuracy holds for high-rate discharge and cranking alike pulses. The model, which is used as an example to show the validity of the approach, needs to be extended to capture the charging behavior.

Tests using identification windows of $4 \mathrm{~min}$ and validation over 60 min show that the battery behavior during HRPSOC use, involving rapidly alternating charge-discharge cycles, does not change. The presented battery model and its established parameters capture the behavior during the whole $60 \mathrm{~min}$.

However, the requirements for a suitable identification window are not revealed. Parameter optimization using windows of the same length containing similar data resulted in different prediction qualities according to two criteria.

\section{ACKNOWLEDGMENT}

The authors would like to thank E. Karden, H. Jung, and E. Aneke (Ford Research Center Aachen) for sharing knowledge and making it possible to carry out experiments with the Ford Fiesta technology demonstrator vehicle.

\section{REFERENCES}

[1] E. Meissner and G. Richter, "The challenge to the automotive battery industry: The battery has to become an increasingly integrated component within the vehicle electric power system," J. Power Sources, vol. 144, no. 2, pp. 438-460, Jun. 2005.

[2] M. Koot, J. Kessels, B. De Jager, W. Heemels, P. Van den Bosch, and M. Steinbuch, "Energy management strategies for vehicular electric power systems," IEEE Trans. Veh. Technol., vol. 54, no. 3, pp. 771-782, May 2005. 
[3] E. Karden, P. Shinn, P. Bostock, J. Cunningham, E. Schoultz, and D. Kok, "Requirements for future automotive batteries-A snapshot," J. Power Sources, vol. 144, no. 2, pp. 505-512, Jun. 2005.

[4] P. Moseley, "High rate partial-state-of-charge operation of VRLA batteries," J. Power Sources, vol. 127, no. 1/2, pp. 27-32, Mar. 2004.

[5] E. Karden, S. Ploumen, B. Fricke, T. Miller, and K. Snyder, "Energy storage devices for future hybrid electric vehicles," J. Power Sources, vol. 168, no. 1, pp. 2-11, May 2007.

[6] B. Bhangu, P. Bentley, D. Stone, and C. Bingham, "Nonlinear observers for predicting state-of-charge and state-of-health of lead-acid batteries for hybrid-electric vehicles," IEEE Trans. Veh. Technol., vol. 54, no. 3, pp. 783-794, May 2005.

[7] D.-T. Lee, S.-J. Shiah, C.-M. Lee, and Y.-C. Wang, "State-of-charge estimation for electric scooters by using learning mechanisms," IEEE Trans. Veh. Technol., vol. 56, no. 2, pp. 544-556, Mar. 2007.

[8] D. Sauer, E. Karden, B. Fricke, H. Blanke, M. Thele, O. Bohlen, J. Schiffer, J. Gerschler, and R. Kaiser, "Charging performance of automotive batteries-An underestimated factor influencing lifetime and reliable battery operation," J. Power Sources, vol. 168, no. 1, pp. 22-30, May 2007.

[9] S. Buller, "Impedance-based simulation models for energy storage devices in advanced automotive power systems," Ph.D. dissertation, RWTH, Aachen, Germany, 2002.

[10] S. Buller, M. Thele, E. Karden, and R. De Doncker, "Impedancebased non-linear dynamic battery modeling for automotive applications," J. Power Sources, vol. 113, no. 2, pp. 422-430, Jan. 2003.

[11] I. Podlubny, Fractional Differential Equations: An Introduction to Fractional Derivatives, Fractional Differential Equations, to Methods of Their Solution and Some of Their Applications. London, U.K.: Academic, 1999.

[12] M. Usman Iftikhar, D. Riu, F. Druart, S. Rosini, Y. Bultel, and N. Retière, "Dynamic modeling of proton exchange membrane fuel cell using noninteger derivatives," J. Power Sources, vol. 160, no. 2, pp. 1170-1182, Oct. 2006

[13] A. Le Mehaute and G. Crepy, "Introduction to transfer and motion in fractal media: The geometry of kinetics," Solid State Ion., vol. 9/10, pp. 17-30, Dec. 1983

[14] M. Aoun, R. Malti, F. Levron, and A. Oustaloup, "Numerical simulations of fractional systems: An overview of existing methods and improvements," Nonlinear Dyn., vol. 38, no. 1/2, pp. 117-131, Dec. 2004.

[15] A. Oustaloup, F. Levron, B. Mathieu, and F. Nanot, "Frequency-band complex noninteger differentiator: Characterization and synthesis," IEEE Trans. Circuits Syst. I, Fundam. Theory Appl., vol. 47, no. 1, pp. 25-39, Jan. 2000.

[16] M. Thele, S. Buller, D. Sauer, R. De Doncker, and E. Karden, "Hybrid modeling of lead-acid batteries in frequency and time domain," J. Power Sources, vol. 144, no. 2, pp. 461-466, Jun. 2005.

[17] M. Thele, E. Karden, E. Surewaard, and D. Sauer, "Impedance-based overcharging and gassing model for VRLA/AGM batteries," J. Power Sources, vol. 158, no. 2, pp. 953-963, Aug. 2006

[18] M. Thele, J. Schiffer, E. Karden, E. Surewaard, and D. Sauer, "Modeling of the charge acceptance of lead-acid batteries," J. Power Sources, vol. 168, no. 1, pp. 31-39, May 2007.

[19] J. Moré and S. Wright, Optimization Software Guide, Frontiers in Applied Mathematics. Philadelphia, PA: SIAM, 1993.

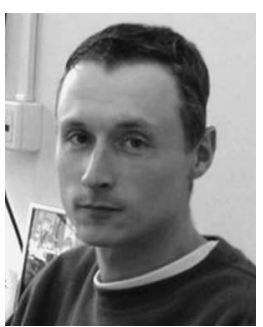

Patrick J. van Bree (S'06) received the M.Sc. degree in electrical engineering in 2006 from Eindhoven University of Technology, Eindhoven, The Netherlands, where he is currently working toward the Ph.D. degree with the Control Systems Group, Department of Electrical Engineering.

His research interests include identification and control of nonlinear systems and their application to industrial problems.

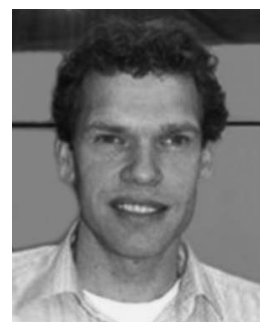

André Veltman (M'95) was born in The Netherlands in 1964. He received the electrical engineering degree from the University of Twente, Enschede, The Netherlands, in 1989 and the Ph.D. degree from the Delft University of Technology, Delft, The Netherlands, in 1994.

From 1986 to 1987, he was a Teaching Assistant with the University of Zambia, Lusaka, Zambia From 1994 to 1997, he was a Postdoctoral Researcher with Eindhoven University of Technology, Eindhoven, The Netherlands, where he worked under a fellowship from the Royal Dutch Academy of Arts and Sciences, Amsterdam, The Netherlands. He spent the first of these three years as a Visiting Postdoctoral Fellow with the University of Wisconsin, Madison, where he worked on flux-based inverter control. In 1997, he founded the design company "Piak Electronic Design" for drive and control systems ranging from watts until megawatts. He is currently a part-time Assistant Professor with Control Systems, Department of Electrical Engineering, Eindhoven University of Technology. He is the author of more than five patents on various subjects such as class-D amplifier control, efficient three-phase current control, paralleling induction motors, sensorless control of linear PM machines, low-cost induction motor controllers, and an acoustic estimator of gas-bottle contents. His main fields of interest are power electronics, class-D amplifiers, frequency inverter control using "The Fish Method," online parameter estimation, highperformance drives, hybrid vehicles, sensorless control, and microcontrollerbased systems.

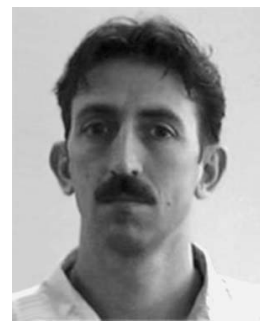

Will H. A. Hendrix received the Bachelor's degree in electrical engineering from Hogere Technische School (HTS) Heerlen, The Netherlands, in 1986.

Since 1987, he has been with the Control Systems Group, Department of Electrical Engineering, Eindhoven University of Technology, Eindhoven, The Netherlands, where he was appointed to the Image Processing Team that was starting up at that time. Later, he became more involved in control systems, and since then, he has been responsible for several laboratory trainings in control. He specializes in realizing prototyping environments at industrial sites for real-time control involving sensors, signal conditioning, data acquisition, and controller implementation. He is responsible for the coordination of several application-oriented research projects involving industries both on a European and national level.

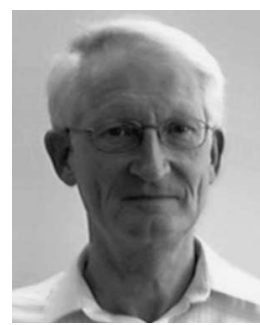

Paul P. J. van den Bosch (M'84) was born in Rotterdam, The Netherlands. He received the M.S. degree in electrical engineering and the Ph.D. degree from the Delft University of Technology, Delft, The Netherlands. His Ph.D. thesis was on "Short term optimization of thermal power systems."

In 1988, he was a full Professor of control engineering with the Delft University of Technology. He has been the appointed Measurement and Control Chair with the Department Electrical Engineering, Eindhoven University of Technology, Eindhoven, The Netherlands, since 1993, and he has also been with the Department of Biomedical Engineering since 2003. He has authored about 150 scientific publications and supervised about 250 M.S. and $30 \mathrm{Ph} . \mathrm{D}$. students. His main research interests deal with modeling and control issues and real industrial products and processes, with considerable experience in electromechanical systems and embedded systems, such as the interface between computer science and control, automotive applications, and biomedical processes. 